\title{
Desafiando a Gravidade: da estática à dinâmica, de objetos a sistemas.
}

\author{
Gonçalo Castro Henriques \\ Universidade Federal Rio de Janeiro, LAMO, PROURB \\ gch@ufrj.br \\ Andrés Passaro \\ Universidade Federal Rio de Janeiro, LAMO, PROURB \\ andrespassaro@fau.ufrj.br
}

\begin{abstract}
In the cold space of information objects drift in the void. All is abandoned in junk space or fiercely organized. The difference between an object as a form and formations - or interacting populations of elements in an environment - is still disregarded. In Cartesian space objects are preconceived by an external observer, in static and timeless space. In parametric space Euclidian geometries persist as preconfigured objects. The flux of information between physical and digital realities is still archaic: is done with effort, demanding physical strength and time. There is low interaction between physical and digital realities. Seems like a dystopian reality in a past-future.

The theory of relativity asserts that space and time are interdependent, being modified and modifying us. In organic and synthetic biology, form results from the dynamic interaction of elements. This Research argues that form and environment are interdependent in physical and digital dimensions. A two-part applied research method is proposed: preliminary research and further development via workshop. Preliminary research identified the knowledge areas involved, defined problem search space and tested methods and techniques to solve it. The successfully developed design methodology - combining the preliminary research and workshop - can be used to solve future design problems.
\end{abstract}

Keywords: Responsive systems; Physical and digital interaction; Algorithmic design; Applied research

\section{Motivação e contexto}

Há muito tempo que o homem desafia a gravidade construindo. As novas tecnologias trazem novas possibilidades técnicas e processos para construir, mas também novos conceitos.

Para superar a pura instrumentalização em projeto, foi proposta uma pesquisa aplicada, dívida em duas partes. Primeiro foi realizada uma análise preliminar sobre o tema, que foi testada num segundo momento através de um workshop. Esta pesquisa continua o desafio de superação da técnica pela técnica, iniciado no workshop anterior «abrigos sensíveis» (Passaro e Henriques 2015). Neste workshop designado como «desafiando a gravidade», o LAMO e um grupo de tutores convidados definiram o contexto de projeto, e conduziram os participantes no desenvolvimento e materialização de um projeto responsivo.

«Desafiando a gravidade» desafiou os participantes a conceber e materializar sistemas responsivos. Começou por exemplificar como se pode passar de uma visão objetual da forma isolada, para uma visão da forma como parte de um sistema. Foram apresentadas aplicações deste princípio utilizando mecanismos simples e mostrando como esses podem interagir com o usuário. Foi oferecida formação aos participantes em projeto assistido por computador (CAAD), fabricação digital (CAM) e interação digital (Arduíno). Estes instrumentos são necessários para conceber, fabricar e operar uma estrutura responsiva.

\section{Problema e metodologia}

Para obter edifícios responsivos é necessário considerar a interação entre a forma, informação e ambiente. A passagem de objetos isolados para sistemas está relacionada com a evolução da física, da matemática e com a transição da causalidade restrita e determinística, para a multicausalidade e o raciocínio probabilístico. O estudo destes fenómenos exige para além de conhecimentos teóricos, conhecimentos práticos. As soluções dependem das técnicas e dos processos utilizados, mas também de como são formalizados, em que escala e em que material.

A pesquisa preliminar procurou ultrapassar estas dificuldades sistematizando o problema e identificando métodos e técnicas, definindo o espaço de busca de soluções. Durante a pesquisa preliminar foi desenvolvida a metodologia do workshop, o que demorou aproximadamente 2 meses, contando com a contribuição - para além dos autores deste texto - de: Elisa Vianna, Rebeca Estrada, Clarice Rohde, Carina Carmo, Vitor Sardenberg, Ernesto Bueno, Verônica Natividade, Lucas de Sordi, Daniel Lenz, Marlus Araújo, monitores do LAMO e outros contributos especificados nos créditos. A pesquisa definiu uma base concetual e operativa envolvendo áreas de conhecimento como arquitetura, teoria de sistemas, programação, robótica, interação, mecânica, 
fabricação digital, entre outras. A base concetual e operativa foram conseguidas através da interação do grupo que preparou o evento. A interação superou a soma das capacidades individuais.

\section{Fundamentação teórica e tecnológica}

$\mathrm{Na}$ pesquisa preliminar foram definidos os fundamentos teóricos e práticos (ferramentas e processos) para desenvolver sistemas responsivos.

\section{Forma (informação) e ambiente}

A definição geométrica utilizada influencia a maneira como projetamos. Os processos digitais e computacionais suportam novos tipos de geometrias, com base em novas definições matemáticas.

Tabela 1: O quadro abaixo, sintetiza as diferenças entre o referencial cartesiano e o topológico (Henriques, 2015).

\begin{tabular}{|ll|}
\hline Referencial cartesiano & Referencial topológico \\
\hline geometria euclidiana & geometria associativa \\
\hline elementos discretos & multiplicidade paramétrica \\
\hline forma ideal regular & forma contínua fluída \\
\hline espaço global infinito & espaço local dimensional \\
\hline espaço neutro & espaço relacional forças \\
\hline tempo contínuo (newton) & tempo simultâneo (einstein) \\
\hline operações isométricas & transformações topológicas \\
\hline
\end{tabular}

A conceção do espaço e da forma euclidiana é diferente da geometria e espaço hiperbólico, ou do espaço paramétrico. A tradução desta geometria em forma física depende das ferramentas que utilizamos e como as utilizamos. A interação entre objetos, depende como são definidas as suas relações e como são representados. A evolução tecnológica é um desafio para reformular o projeto.

\section{Da forma para formações, de ambientes estáticos para dinâmicos}

A visão objetual da forma como objeto isolado ou como conjunto articulado de partes está associada ao desenvolvimento do conceito sistema. No workshop foi apresentado um resumo sobre sistemas responsivos, que aqui é sintetizado.

A Teoria Geral dos Sistemas e a disciplina da cibernética trouxeram novos contributos matemáticos para a definição de sistema. A Teoria Geral dos Sistemas - desenvolvida Ludwig Von Bertalanffy (1952-1968) - representa uma reação ao reducionismo e causalidade restrita, promovendo o holismo e a multicausalidade. Esta teoria promoveu a visão de organismo, em que o todo é maior que a soma das partes. Desenvolveu também, leis matemáticas gerais de sistemas - sejam eles naturais, artificiais, sociais, etc. (De Landa 2011). Gordon Pask contribui, nesta área, com o desenvolvimento da teoria do controlo dos sistemas, a cibernética (1969).
Durante o workshop foi explicada a importância da definição de mecanismo simples de feed-back apresentada por Von Bertalanffy e a diferença para o mecanismo de adaptação orientado por objetivos apresentado por Gordon Pask.

Desde a definição geométrica de objetos euclidianos num espaço infinito, até à geometria topológica de campos, há uma evolução. O desenvolvimento da matemática permitiu definir relações associativas entre elementos geométricos. Se no referencial cartesiano cada objeto é definido de maneira isolada no espaço infinito, num espaço topológica pelo contrário podemos definir geometrias associativas em espaços locais. Por exemplo podemos definir superfícies tipológicas compostas por componentes, relacionados entre si, formando um sistema. Assim as formas podem variar de acordo com o referencial e a definição matemática utilizada. A física newtoniana considera os objetos separadamente, mas com a evolução de conceitos e instrumentos foi possível passar a considerar campos de forças, definidos pela interpolação entre as suas partes. É possível passar de uma definição da forma num referencial neutro e estático, para a definição interativa da forma num ambiente relacional dinâmico, ou seja através de definições geométricas mais avançadas é possível considerar o meio tanto como resultado, como resultante da interação entre as partes.

\section{Pesquisa aplicada preliminar}

\section{Diagramas de interação}

Para além da recolha teórica de exemplos foi necessário encontrar um método para testar a pesquisa teórica. Para desenvolver sistemas é necessário definir a interação das partes com o meio. Procurou-se capturar a interação entre a(s) forma(s) e determinados movimentos, traduzindo esta interação graficamente através de diagramas. A ideia de utilizar diagramas generativos foi desenvolvida no workshop anterior «abrigos sensíveis». Estes diagramas foram sendo discutidos e aperfeiçoados pelo grupo de preparação, num processo interativo. A análise sobre os tipos de interação permitiu definir categorias de movimentos e aperfeiçoar a sua representação gráfica. Os diagramas potenciaram o desenvolvimento de mecanismos físicos e digitais. Pensouse na tradução física e analógica através de transmissões (engrenagens); foram desenvolvidos mecanismos digitais através de algoritmos. A ligação entre os mecanismos físicos e digitais foi desenvolvida através de sensores e atuadores. Com o desenvolvimento associado prévio de software e hardware, procurou-se minimizar a separação físico-digital. Os diagramas desenvolvidos sintetizam uma ação (verbo), mas é necessário saber como ele se processa. De entre os diagramas desenvolvidos foram escolhidos os diagramas identificados pelos seguintes verbos: despregando, levantando, transladando, desdobrando e enrolando.

A tradução dos diagramas permitiu pesquisar soluções e identificar dificuldades. A pesquisa algorítmica foi coordenada e desenvolvida por Gonçalo Henriques, Ernesto Bueno e Verônica Natividade. Nesse processo foram pensados exercícios de programação relacionados com os objetivos de projeto. Não se tratou de antecipar soluções, 
mas antes de estudar maneiras de solucionar problemas através de diferentes algoritmos. Este procedimento difere da maioria dos workshops sobre programação em que a aprendizagem se baseia na resolução de exercícios genéricos. A tradução dos mecanismos físicos e analógicos foi desenvolvida com o Lucas de Sordi, Daniel Lenz e Marlus Araújo. Este desenvolvimento permitiu mapear tipos de mecanismos e o material necessário para o workshop. Os diagramas de movimento permitiram desenvolver algoritmos, mecanismos físicos (materiais) e a transmissão de
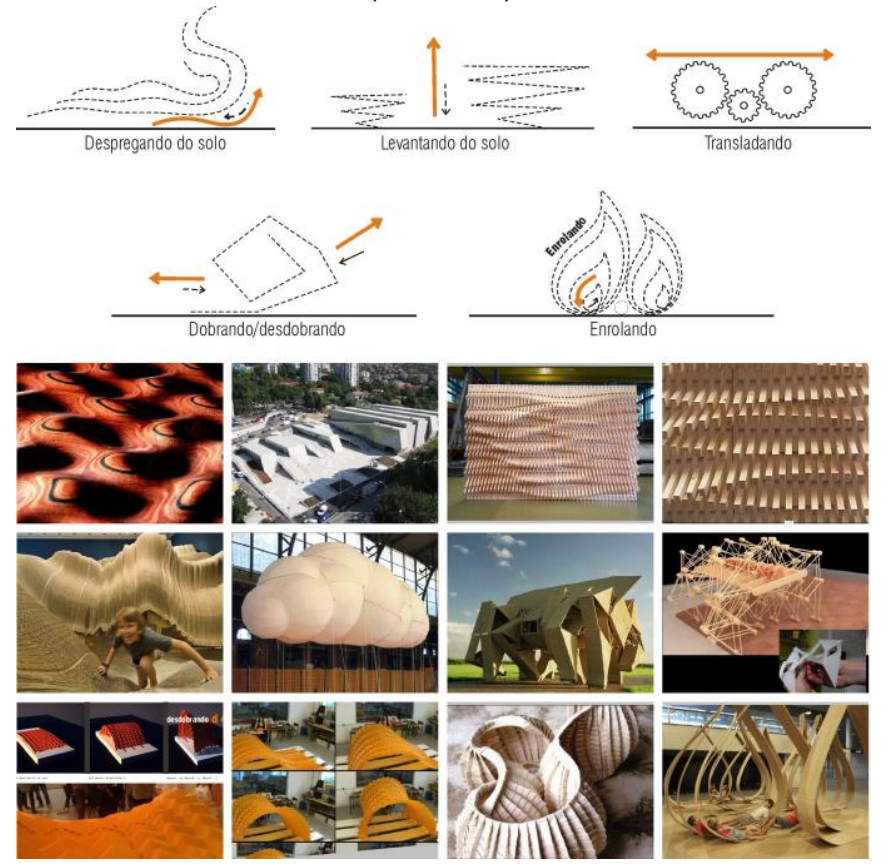

informação entre ambos.

Figura 1: Em cima Diagramas de movimento do solo sugeridos aos grupos: despregando, Levantando, transladando, dobrando, enrolando. Em baixo imagens de projetos de referência que são ou tem potêncial para ser responsivos, da esquerda para a direita, de cima para baixo: Bernhard Cache (Fonds 1999), 3LHD (Zemet Center Rijeka, 2009), Gramazio \& Kohler (Sequential wall, 2008), Ball Nogues (Rip Curl Canyon, 2006), The cloud H1(Bélgica, desconhecido), Theo Jansen, (Rhinoceros, 1999), Filipa Osorio (KOS, 2014), Christine Edison (Mother and Child', 2009), Caterina Tiazzoldi (Onion Pinch, Lisboa 2009), fonte das imagens internet.

Para testar esta metodologia o grupo procurou exemplos de construções sobre o solo que se modificassem a sua forma para interagir. A pesquisa identificou superfícies compostas por componentes dinâmicos. No entanto a maioria dos exemplos identificados, apesar de estáticos possuíam um potencial elevado de se tornarem interativos. Os diagramas foram desenvolvidos procurando exemplos construídos e vice-versa, como explicado na figura 1. Este tipo de formas que ainda que sendo estáticas têm um potencial dinâmico remete para um livro de referência sobre vitalismo da forma "animated form" (Lynn, 1999). Para transformar as formas estáticas em dinâmicas recorreu-se ao conceito de mecanismo, que é detalhado a seguir

\section{Mecanismos e sistemas emergentes}

A articulação entre as partes de um sistema é feita através de mecanismos. Holland define um mecanismo como um dispositivo que responde a ações (input ou entrada informação) processando esse input, produzindo ações como resultado (output ou saída de informação). Esta definição está relacionada com a definição de sistema proposta por Bertalanffy. Holland (1999) providencia uma descrição de mecanismos citando exemplos desde os gregos (alavancas simples), passando pelos árabes (relógios e mecanismos dentados) até aos processos computacionais (Jogo da vida Conway).
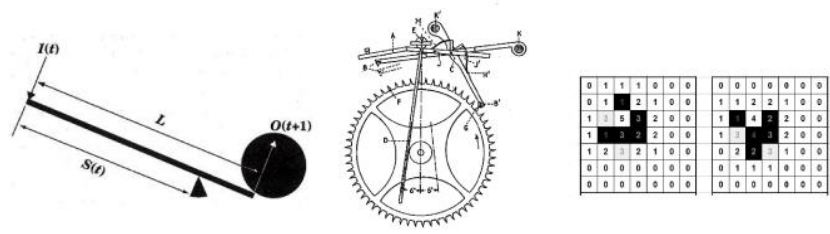

Figura 2: Mecanismos: alavanca definida parametricamente (baseado em Holland 1999); representação geométrica de pêndulo e mecanismo gafanhoto de relógio (baseado em Harrison 1713 ); autómato celular Conway baseado em regras de estados(adaptado de Conway 1999).

Os mecanismos apresentados na figura têm diferentes lógicas e representações. O primeiro mecanismo é o mecanismo da alavanca, que já existe desde os gregos, mas é traduzido numa fórmula que adquire vários resultados (ou estados) de acordo com os parâmetros que lhe são atribuídos. O segundo mecanismo está associado à descrição das órbitas esféricas dos pêndulos por Huygens (Séc. XVII); o pêndulo está descrito geometricamente, sendo o seu mecanismo baseado no movimento gerado pelo peso próprio de um pêndulo acionando um mecanismo "tipo gafanhoto" que movimenta uma roda dentada de um relógio analógico. O terceiro mecanismo é baseado em autómatos celulares, que traduz regras analógicas em regras matemáticas digitais, com resultados emergentes.

Os materiais possuem também regras que tornadas explícitas podem ser traduzidas em mecanismos com estados que podem gerar resultados emergentes. Um exemplo da utilização desta metodologia é o trabalho de pesquisa de Achim Menges que fundou o Instituto de Design Computacional na Universidade de Stuttgart em 2009. Neste workshop foram mostrados sistemas materiais existentes, compostos por componentes simples que apesar de imóveis apresentavam um potencial para assumir diferentes estados, antevendo o desenvolvimento associado de mecanismos. Como referência de geração da forma foi citada a teoria da emergência dos sistemas de que falam entre outros (Holland 1998 e Weinstock, 2004 em que através de simples condições locais se podem gerar processos complexos, em que as possibilidades de comportamento não podem ser aferidas pelas propriedades das partes, em que o resultado da interação supera as propriedades dessas partes separadas. Weinstock destaca o fundo matemático desta 
teoria e Holland mostra como utilizar estes princípios em computação

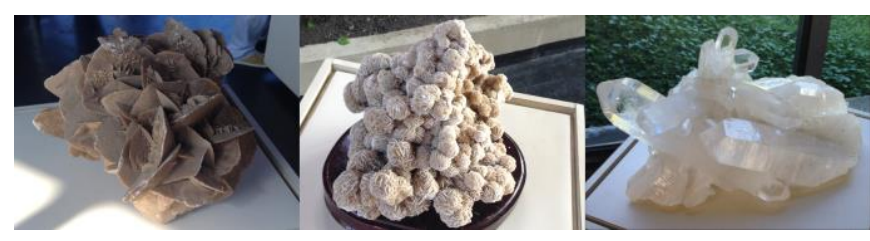

Figura 3: Regras de formação de materiais: exemplos de formações geométricas naturais de minerais resultantes de processo físicoquímicos. CETEM, UFRJ, Fotos Gonçalo Henriques.

\section{Desenvolvimento do workshop}

O workshop foi pensado para 8 dias com 5 tutores, sendo selecionados 20 participantes. Nos primeiros dias houve palestras teóricas com formação simultânea em design algorítmico (grasshopper), fabricação digital, redes interativas (sensores e Arduino) e analógico-digitais (forças e movimentos).

Os participantes foram divididos em 4 grupos, tendo escolhido como ponto de partida um dos 5 diagramas acima descritos. Cada projeto procurou associar ao diagrama um movimento e um estímulo, definido um princípio de açãoreação. Os estímulos utilizados foram a presença humana, luz, som, magnetismo, etc. Além do estímulo, foi pedido que se identificasse 0 estímulo inverso e se refletisse no processo de reajuste: se a luz provoca a abertura de uma forma, a sua ausência deverá provocar o seu fechamento. A interação com o utilizador depende de propriedades físicas como densidade/leveza, permeabilidade/opacidade, capacidade agregação/expansão e da interação das partes em conjunto.

Foram propostas a seguintes etapas de desenvolvimento de projeto: 1- Diagrama de movimento; 2- definição de Geometria/Materialidade, definindo os componentes/primitivas e o material; 3- definição do estímulo associado ao diagrama (luz, som, humidade, temperatura); 4- definição de um pseudo código do processo de interação. Cada grupo concebeu um sistema e produziu um modelo funcional analógico-digital de uma superfície responsiva com uma dimensão máxima de 50×50×50 cm.

\section{Algoritmos e programação visual}

Foram pensados vários exercícios de programação para os alunos, relacionados com os sistemas que foram depois desenvolvidos, como o da Figura 4. Esta preparação também fez parte da pesquisa preliminar.

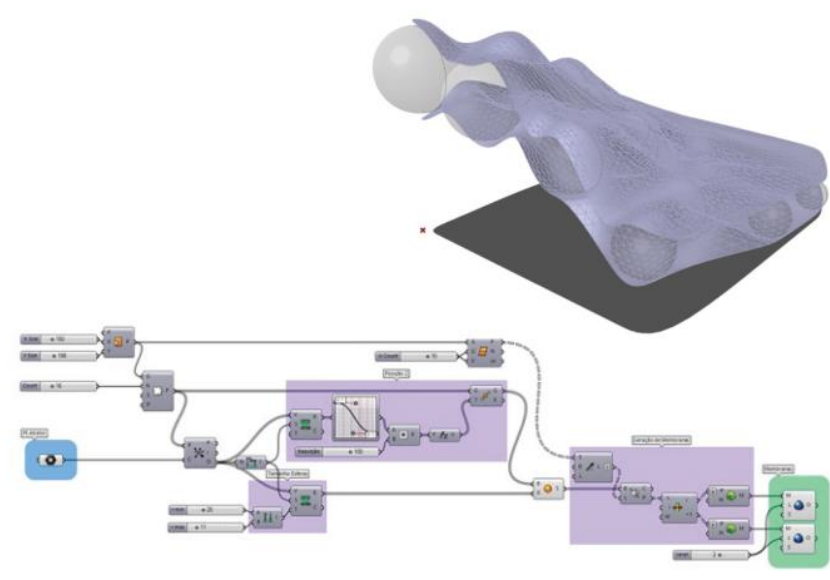

Figura 4: Algoritmo e modelo de membranas deformadas por esferas afetadas por um atrator. Desenvolvimento: Bueno e Henriques, imagem de Ernesto Bueno.

\section{Resultados workshop}

Os resultados mais palpáveis do workshop são os trabalhos desenvolvidos pelos grupos, que serão agora descritos.

O Grupo1 partiu do movimento despregando. O projeto "labirinto de areia" desenvolveu uma paisagem que se modifica de acordo com a proximidade humana. Os estímulos são captados através de sensores de movimento, que são o input de mecanismo de software, que através de um conjunto de estados geram valores que são aplicados em diferentes locais da superfície que se altera. Um dos maiores desafios foi definir a geometria do solo para encontrar lógicas dos materiais e dos componentes que potenciassem 0 movimento da superfície como um todo. Foram discutidas vantagens de utilizar malhas poligonais (poligonal meshes) e superfícies NURBS, com diferentes definições matemáticas.

O Grupo2 partiu do movimento levantando, definindo a "chuva" um sistema de partículas que reage à presença humana. Foi definido um processo para controlar uma nuvem de componentes através de um só ponto. Foi desenvolvido um mecanismo analógico para controlar a nuvem de pontos através de um ponto central distribuído em 4 colunas, puxado por 4 motores. Os pontos funcionam como uma casca convexa, definindo a variação das equações dos pontos diferentes curvaturas que variam de acordo com um conjunto de estados que variam de acordo com a posição do usuário. A distância do usuário é transmitida através de sensores para uma placa arduíno que passa o sinal para o mecanismo digital que calcula a posição dos componentes e que devolve a posição e aciona o sistema de motores para a posição pretendida. Foi conseguido um sistema com um movimento elegante e fluido analógico-digital.

O Grupo 3 foi enrolando o solo como uma planta reagindo à alteração de luz, numa "Neo-nastia". Esta planta analógicodigital recebe input de sensores de luz e através do algoritmo desenvolvido altera a sua forma enrolando-se sobre si mesma. Segue uma órbita incremental de acordo com a 
sequência de Fibonacci, que está presente no crescimento e
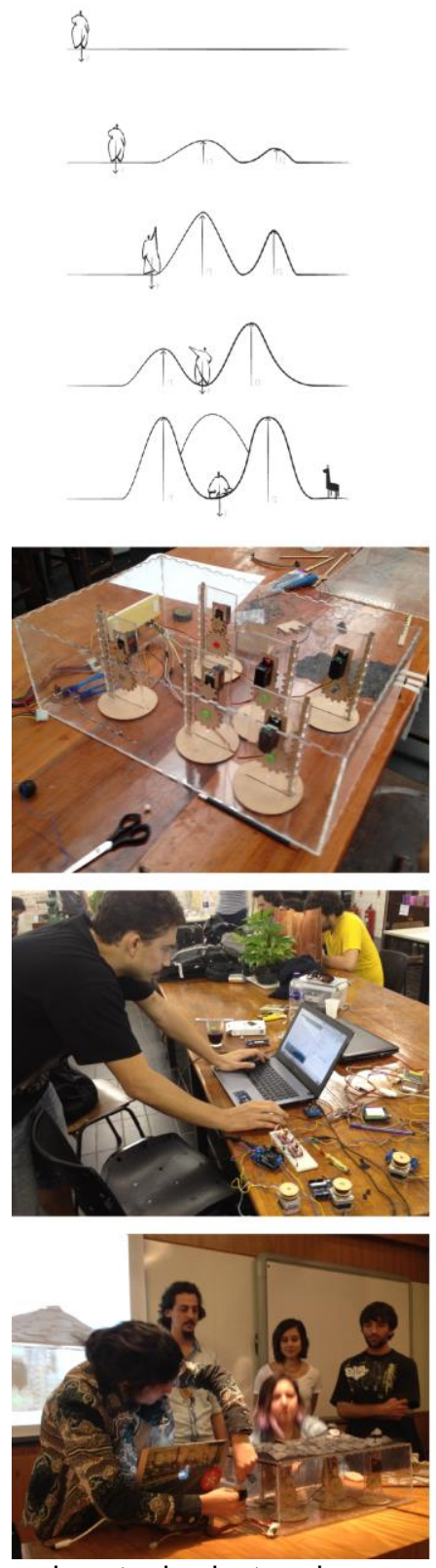

movimento de plantas vivas.
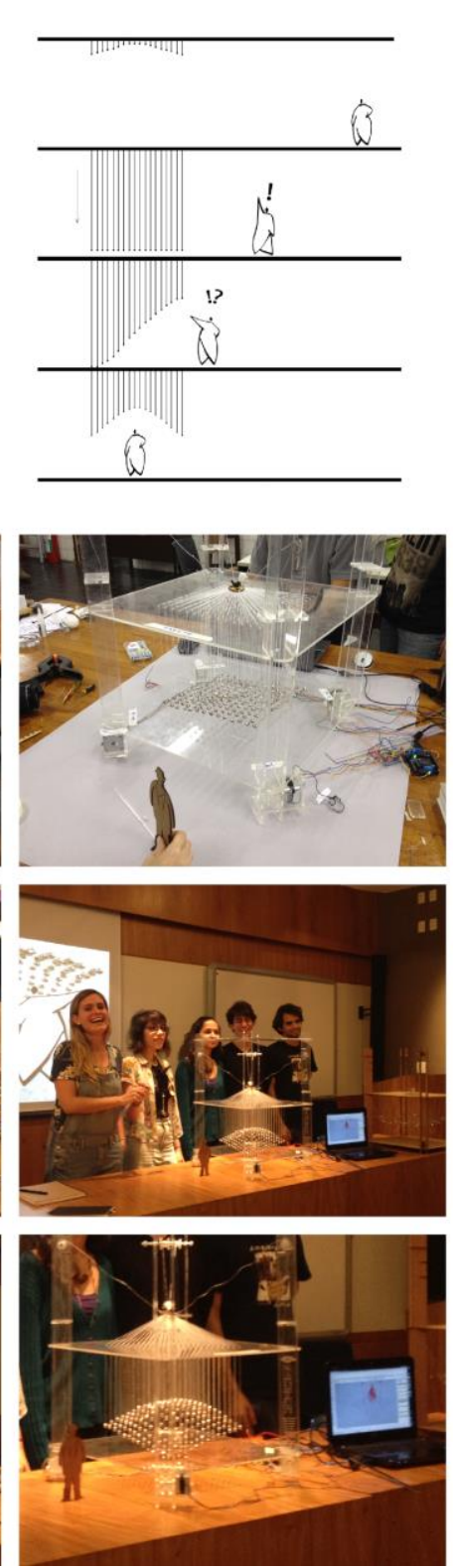
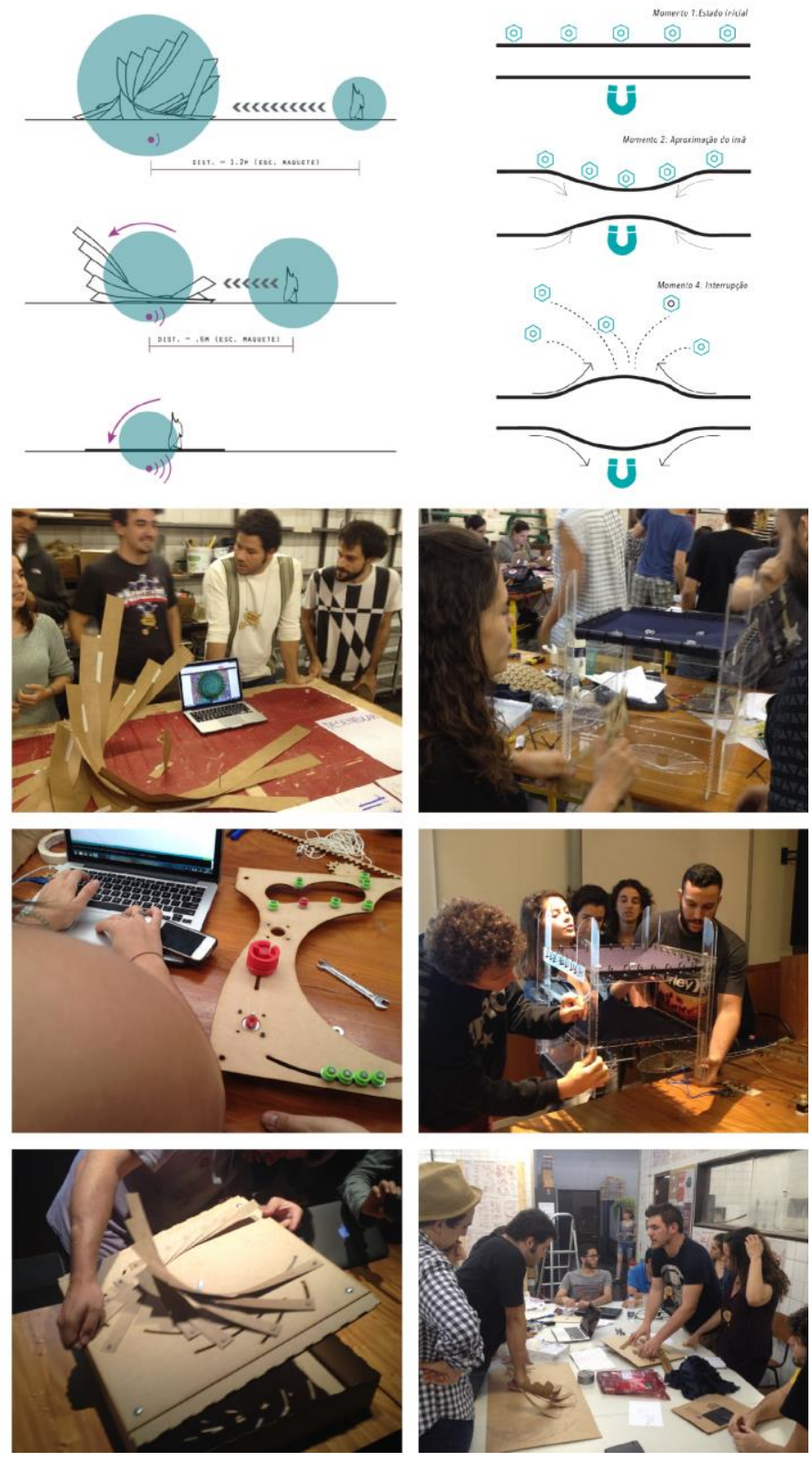

Figura 5: Cada grupo do workshop partiu de um diagrama de movimento do solo e criou uma narrativa de projeto, desenvolvendo um sistema responsivo. Trabalho dos grupo da esquerda para direita G1. G2, G3 e G4, fotografias Gonçalo Henriques.

O Grupo 4 desdobrando o solo definiu um espaço

dimensional de duas superfícies que se juntam criando o designado «buraco de minhoca». Utilizando um touch-pad é modificada a articulação de duas superfícies, primeiro na definição paramétrica. Depois a informação é transmitida a um mecanismo de rodas dentadas que reproduz a forma no modelo físico, resultando no movimento de um íman que causa a atração ou repulsão de componentes metálicos existentes na superfície através de estímulos magnéticos. 


\section{Discussão metodologia aplicada}

Desde que começou a construir, o homem utilizou a tecnologia para criar um novo solo. Organizou o espaço, estabelecendo uma nova ordem, aparentemente em contraste com a desordem natural. Vitruvius incluiu as máquinas da guerra no seu tratado de arquitetura. No entanto há muito que a natureza é estudada como uma máquina. Com o desenvolvimento da teoria dos sistemas e cibernética passou-se a considerar a interação dos sistemas naturais ou artificiais. O desafio do workshop foi definir superfícies mutáveis, constituídas por conjuntos de objetos que interagissem no espaço e no tempo com o homem. $O$ material escolhido e a geometria influenciam a relação destas superfícies com o contexto, mas os seus mecanismos de software também. Estes mecanismos atribuem um novo vitalismo à matéria, estas máquinas ganham animismo. Considerando que a maioria dos participantes não tinha experiência anterior, os resultados deste workshop são promissores. Estes resultados sugerem também que a metodologia desenvolvida - identificando referências teóricas e práticas através de experimentos preliminares e testada depois na pratica - pode ser utilizada para resolver outros problemas de projeto

\section{Apoio à pesquisa}

CNPq, CAPES, FAPERJ, PROURB e LAMO.

\section{Créditos Workshop}

Coordenação Workshop: Andrés Passaro, Gonçalo Castro Henriques e Maria Elisa Vianna. Comité Científico: Andrés Passaro/FAU-UFRJ, Gonçalo Castro Henriques/FAU-UFRJ, Guto Nóbrega/EBA-UFRJ, Malú Fragoso/EBA-UFRJ, Carlos Feferman/FAU-UFRJ, Naylor Villas Boas/FAU-UFRJ, Rodrigo Cury/FAU-UFRJ, Verônica Natividade/PUC-Rio. Organização: Carina Carmo, Clarice Rohde, Maria Elisa Vianna, Rebeca Duque Estrada. Colaboração: Victor Sardenberg. Palestras: Andrés Passaro, Arthur Lara, Carina Carmo, Clarice Rohde, Daniel Lenz, Ernesto Bueno, Gabriela Celani, Gonçalo Castro Henriques, Guto Nóbrega, Laura Lago Basile, Rebeca Duque Estrada, Verônica Natividade.

Tutores: Ernesto Bueno, Gonçalo Castro Henriques e Verônica Natividade (Programação), Daniel Lenz, Lucas de Sordi e Marlus Araújo (Interação). Participantes: Aline Furtado, Anael Alves, Bernardo Luís do Amaral, Branca Leibovich, Caio Cavalcanti, Camila Rodriguez, Camila Torres, Carlos Zebulun, Cíntia Mechler, Dimmy Trindade, Gabriel Gaspar, Gabriel Raymundo, Gabriela Bonifacio, Isadora Tebaldi, Lia Guerra, Lina Lopes, Loan Tammela , Luciano Faria, Maria Rúbia Pereira, Marinah Raposo, Raquel Leite, Roberto da Matta, Thomaz Vieira, Vanessa Rosa. Fotografia: Ana Carolina Moreno, Anna Carolina Peres, Helena Burock, Júlia Roizemberg, Mariana Maciel, Nicolas Araújo, Yasmim Anefalos. Edição vídeo: Bruno Pavão, Eduardo Sant'anna e Filipi Dias. Ilustrações: Júlia Nodari. Equipe LAMO e suporte: Camila Torres, David Mendonça, Felipe Madeira, Gabriel Gaspar, Helena Burock, Lucas
Fontes, Luciano Faria, Roberto da Matta, Rudá Mafra, Thiers Freire, Vinícius Lucena, Adalberto Pereira Filho, Christiannne Cezar Albuquerque.

\section{Referências:}

ALEXANDER, Christoph, "System Generating systems", em Architectural Design, December Issue No 7/6, John Willey \& Sons Ltd, Londres 1991, pp. 90-1.

BERTALANFFY, Ludwig Von, General System Theory: Foundations, Development, Applications, George Braziller, Nova lorque, 1969.

DELANDA, Manuel, Real Virtuality, em "Computational Design Thinking", MENGES, Achim, AHLQUIST, Sean (eds), John Willey Sons Ltd, Chichester, Reino Unido, 2011.

HENRIQUES, Gonçalo Castro; "Sistemas responsivos: relevância, estado da arte e desenvolvimentos", XIX SIGraDi, Informação Projeto para interação, Alice Pereira e Regiane Pupo (eds), Blücher, pp. 200-6, DOI 10.5151/despro-sigradi2015-60042

HOLLAND, John, Constrained Generating Procedures, Emergence: From Chaos to Order, Perseus Books (Reading, MA), 1998, pp 125- 42.

LYNN, Greg, Animated Form, Princeton Architectural Press, New York, 1999.

MENGES, Achim, AHLQUIST, Sean, Computational Design Thinking, John Willey Sons Ltd, Chichester, Reino Unido, 2011.

PASK, Gordon "The Architectural Relevance of Cybernetics", em Architectural Design, September issue No $7 / 6$, John Wiley \& Sons, Londres, 1969.

PASSARO, Andrés; HENRIQUES, Gonçalo Castro; "Abrigos Sensíveis, do método ao conceito, superando a instrumentalização", XIX Congresso SIGraDi, Informação Projeto para interação, Alice T. PEREIRA e Regiane Pupo (eds), Blucher Publishing, pp. 94-100, DOI 10.5151/despro-sigradi2015-30155.

WEINSTOCK, Michael, Emergence: Morphogenetic Design Strategies in Emergence: Morphogenetic Design Strategies, HENSEL, Michael MENGES, Achim WEINSTOCK, Michael (eds), Academy Press; Londres 2004, pp. 6-14. 